\title{
Genetic Investigation of Complement Pathway Genes in Type 2 Diabetic Retinopathy: An Inflammatory Perspective
}

\author{
Ming Ming Yang, ${ }^{1,2}$ Jun Wang, ${ }^{3}$ Hong Ren, ${ }^{4}$ Yun Duan Sun, ${ }^{1}$ Jiao Jie Fan, ${ }^{1}$ \\ Yan Teng, ${ }^{1}$ and Yan Bo $\mathrm{Li}^{3}$ \\ ${ }^{1}$ Eye Hospital, The First Affiliated Hospital of Harbin Medical University, Harbin 150001, China \\ ${ }^{2}$ The Centre for Endemic Disease Control, Chinese Center for Disease Control and Prevention, Harbin Medical University, \\ Harbin 150001, China \\ ${ }^{3}$ Department of Endocrinology, The First Affiliated Hospital of Harbin Medical University, Harbin 150001, China \\ ${ }^{4}$ Operating Room, The First Affiliated Hospital of Harbin Medical University, Harbin 150001, China
}

Correspondence should be addressed to Ming Ming Yang; ming4622@163.com

Received 16 November 2015; Revised 10 January 2016; Accepted 12 January 2016

Academic Editor: Joilson O. Martins

Copyright (C) 2016 Ming Ming Yang et al. This is an open access article distributed under the Creative Commons Attribution License, which permits unrestricted use, distribution, and reproduction in any medium, provided the original work is properly cited.

\begin{abstract}
Diabetic retinopathy (DR) has complex multifactorial pathogenesis. This study aimed to investigate the association of complement pathway genes with susceptibility to DR. Eight haplotype-tagging SNPs of SERPING1 and C5 were genotyped in 570 subjects with type 2 diabetes: 295 DR patients (138 nonproliferative DR [NPDR] and 157 proliferative DR [PDR]) and 275 diabetic controls. Among the six C5 SNPs, a marginal association was first detected between rs17611 and total DR patients $(P=0.009, \mathrm{OR}=0.53$ for recessive model). In stratification analysis, a significant decrease in the frequencies of G allele and GG homozygosity for rs17611 was observed in PDR patients compared with diabetic controls $\left(P_{\text {corr }}=0.032\right.$, OR $=0.65$ and $P_{\text {corr }}=0.016, \mathrm{OR}=0.37$, resp.); it was linked with a disease progression. A haplotype AA defined by the major alleles of rs17611 and rs1548782 was significantly predisposed to PDR with increased risk of $1.54\left(P_{\text {corr }}=0.023\right)$. Regarding other variants in C5 and SERPING1, none of the tagging SNPs had a significant association with DR and its subgroups (all $P>0.05$ ). Our study revealed an association between DR and C5 polymorphisms with clinical significance, whereas SERPING1 is not a major genetic component of DR. Our data suggest a link of complement pathway with DR pathogenesis.
\end{abstract}

\section{Introduction}

Diabetes mellitus $(\mathrm{DM})$ is reaching an alarming proportion worldwide, as is known that DM has a complex multifactorial pathogenesis. The devastating complications of diabetes are the macro- and microvascular diseases $[1,2]$. Of them, diabetic retinopathy (DR) is the most common microvascular complication and is a leading cause of blindness across the globe [3]. To date, many environmental and clinical factors have been proposed to confer risk of DR development, such as prolonged duration of diabetes, alteration of glucose metabolism, and poor glycemic control [4]. Additionally, genetic predisposition, independent of the above-mentioned factors, has been found to contribute to DR pathology; the evidence comes from the observation of disease aggregation among family members and multiple DR-associated genes identifications [5-7]. So far, the exact pathogenesis of DR is still unclear and is known to be involved in several physiopathologic pathways, such as angiogenesis factors, oxidative stress, apoptosis, and protein kinase C (PKC) [810]. More recent evidence depicting DR as a retinal disease associated with inflammation has drawn special attention and garnered great research interests [11-13].

Complement system is an important component of innate immunity and involved in the modulation of several immune and inflammatory responses. The complement system can be divided into classical, lectin, and alternative pathway; activation of the system is tightly regulated by complement 
factors; disruption of complement regulation can lead to several distinct downstream inflammatory actions en route to the pathogenesis of DR $[14,15]$. Evidence for the link comes from the observation of increased expression of several complement factors in DR patients; these factors included $\mathrm{C} 1$ inhibitor (C1INH, also known as serpin peptidase inhibitor, clade G, SERPING1), C5, factor $\mathrm{H}(\mathrm{CFH})$, and factor B (CFB) $[16,17]$. In our previous studies, genetic variants in the $C F H$ and $C F B$ genes, both involved in complement alternative pathway, have been evaluated and identified as susceptibility genes for DR [18]. Moreover, $C F H$ and $C F B$, as well as other complement pathway genes, have also been found to be associated with a range of inflammatory diseases [19].

Therefore, a genetic study focused on other complement genes was designed with a view to elucidating the involvement of complement system in DR development. Two complement genes, SERPING1 and C5, involved in the classical pathway and in the central part of complement cascade, respectively, were selected for evaluation. Furthermore, stratification by DR stage and genotype-phenotype correlation analysis were also performed to identify these factors associated with prognosis and clinical features.

\section{Materials and Methods}

2.1. Study Participants. The study protocol was approved by the Ethics Committee on Human Research, Harbin Medical University. The study procedures were conducted in accordance with the tenets of the Declaration of Helsinki. Written informed consent was obtained from all study subjects after explanation of the nature of the study. All study subjects were Han Chinese recruited from the First Affiliated Hospital of Harbin Medical University.

All patients received complete ophthalmic examinations and clinical information collection, including corrected visual acuity, slit-lamp biomicroscopy, fundoscopic examination, age, gender, progression time from diabetes to DR, body mass index (BMI), HbAlc level, smoking status, and presence of hypertension and hyperlipidemia, as well as insulin application. The study involved 570 unrelated individuals with type 2 diabetes mellitus (T2DM); patients with type 1 diabetes, gestational diabetes, or maturity-onset diabetes were excluded from the study. The diagnosis of T2DM was based on World Health Organization criteria [20]. Of the group, 295 patients were diagnosed with DR (156 [52.9\%] PDR and 139 [47.1\%] NPDR); 275 subjects without DR but with type 2 diabetes duration of more than 10 years were considered as DM controls. The stage of DR was determined according to the Early Treatment Diabetic Retinopathy Study (ETDRS) criteria [21]. People with any systemic inflammation diseases, or any other ocular disorders such as age-related macular degeneration (AMD), glaucoma, or retinal venous occlusion, were also excluded.

2.2. SNP Selection and Genotyping. We adopted a haplotypetagging SNP approach and obtained the tagging SNPs across the targeted regions, from the International HapMap Project for the Chinese Han Beijing (CHB) population (http://hapmap.ncbi.nlm.nih.gov/, HapMap Genome Browser). Two SNPs (rs1005511 and rs3824988) from SERPING1 and six from C5 (rs12237774, rs2269066, rs17611, rs1548782, rs10985126, and rs1017119) were selected by the taggerpairwise method with $r^{2}$ and MAF (minor allele frequency) values greater than 0.8 and 0.10 , respectively. Genomic DNA was extracted from whole blood using a QIAamp Blood Kit (Qiagen, Hilden, Germany) according to the protocol. All the SNPs were genotyped by TaqMan SNP Genotyping Assays (Applied Biosystems Inc., Foster City, CA) in the LightCycler ${ }^{\circledast}$ 480 Real-Time PCR System (Roche, Switzerland) according to the manufacturer's instructions.

2.3. Statistical Analysis. Hardy-Weinberg equilibrium (HWE) of individual SNP was tested by $\chi^{2}$ test. Allelic and genotypic association of each SNP was calculated by using $\chi^{2}$ test or Fisher exact test. Dominant and recessive models were also applied to investigate the disease association with regard to the minor allele. The odds ratios (OR) and 95\% confidence intervals (CI) were calculated. Pairwise linkage disequilibrium (LD, $D^{\prime}$ ) between polymorphisms and expectation-maximization- (EM-) based haplotype association analysis were assessed using the Haploview software. Student's $t$-test and $\chi^{2}$ test were used to compare continuous clinical data and categorical variables, respectively. Stratification analysis based on DR stage (NPDR and PDR) was also performed. $P<0.05$ was considered as statistically significant. $P$ values were corrected by Bonferroni test $(n=$ total number of SNPs) or permutation test in Haploview software.

\section{Results}

In our study, a total number of 570 unrelated individuals with T2DM were recruited, comprising 295 DR patients and 275 DM controls. Since we aimed to recruit T2DM patients without DR as controls, the mean duration of disease was longer than that of the DR group so as to largely rule out lateonset DR $(P<0.01)$. The proportions of hyperlipidemia and insulin application were higher in DR group than that in DM controls $(P=0.042$ and $P<0.001$, resp. $)$. No significant differences in other clinical features were observed between two groups (Table 1).

Two haplotype-tagging SNPs in SERPING1 and six haplotype-tagging SNPs in C5 were selected, which capture over $90 \%$ of all alleles across their corresponding locus with a MAF larger than 0.10 and a mean $r^{2}$ of 0.80 in the HapMap Chinese Han population. The genotype frequencies of the eight selected SNPs followed the HWE in all subjects. For C5rs17611, there was an obvious trend towards lower proportions of $G$ allele and GG homozygosity in DR patients than DM controls $(P=0.056, \mathrm{OR}=0.79,95 \% \mathrm{CI}=0.62-1.0 ; P=0.009$, $P_{\text {corr }}=0.072, \mathrm{OR}=0.53,95 \% \mathrm{CI}=0.33-0.86$, resp.), but the associations either were marginal or could not remain after adjustment for multiple testing. For other SNPs, no significant associations were detected with DR in any genetic models (Table 2). 
TABLE 1: Characteristics of the study subjects.

\begin{tabular}{|c|c|c|c|}
\hline Characteristic & $\begin{array}{c}\mathrm{DR} \\
(n=295) \\
\end{array}$ & $\begin{array}{c}\mathrm{DM} \\
(n=275) \\
\end{array}$ & $P$ value \\
\hline Age (years) & $55.9 \pm 13.2$ & $56.3 \pm 7.6$ & 0.66 \\
\hline Gender (F/M) & $151 / 144$ & $153 / 122$ & 0.29 \\
\hline Duration of diabetes (years) & $13.1 \pm 9.4$ & $18.1 \pm 6.7$ & $<0.01$ \\
\hline Duration of DR (years) & $5.3 \pm 3.9$ & - & N/A \\
\hline $\begin{array}{l}\text { Progression time from diabetes } \\
\text { to DR (years) }\end{array}$ & $7.9 \pm 5.8$ & - & N/A \\
\hline HbAlC (\%) & $8.0 \pm 1.6$ & $7.9 \pm 1.9$ & 0.50 \\
\hline BMI $\left(\mathrm{kg} / \mathrm{m}^{2}\right)$ & $24.0 \pm 5.7$ & $24.1 \pm 4.4$ & 0.82 \\
\hline Hypertension (\%) & 73.2 & 66.9 & 0.10 \\
\hline Hyperlipidemia (\%) & 30.8 & 23.3 & 0.042 \\
\hline Smoking (\%) & 13.9 & 16.0 & 0.48 \\
\hline Insulin therapy (\%) & 46.8 & 26.2 & $<0.001$ \\
\hline Family history of diabetes (\%) & 26.1 & 21.5 & 0.19 \\
\hline
\end{tabular}

$P$ values were compared by $\chi^{2}$ or Student's $t$-test, and $P<0.05$ was considered statistically significant. DR: diabetic retinopathy; DM: diabetes mellitus; HbAlc: glycosylated hemoglobin; BMI: body mass index.

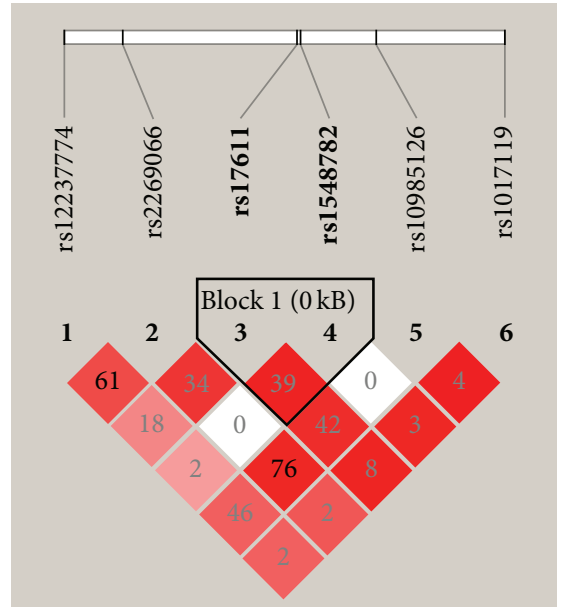

(a)

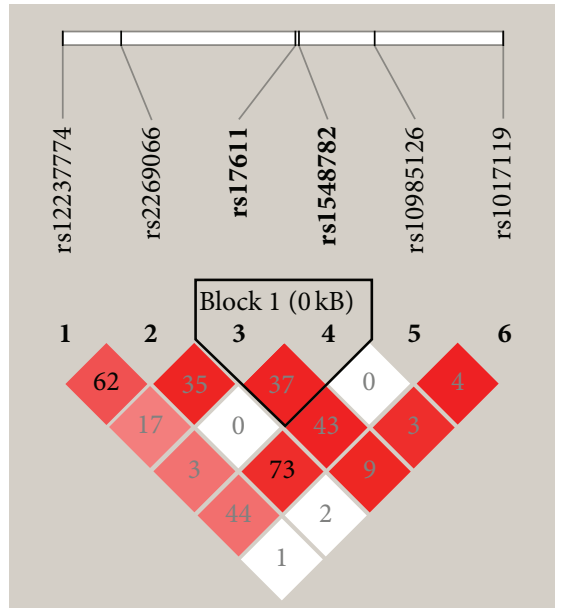

(b)

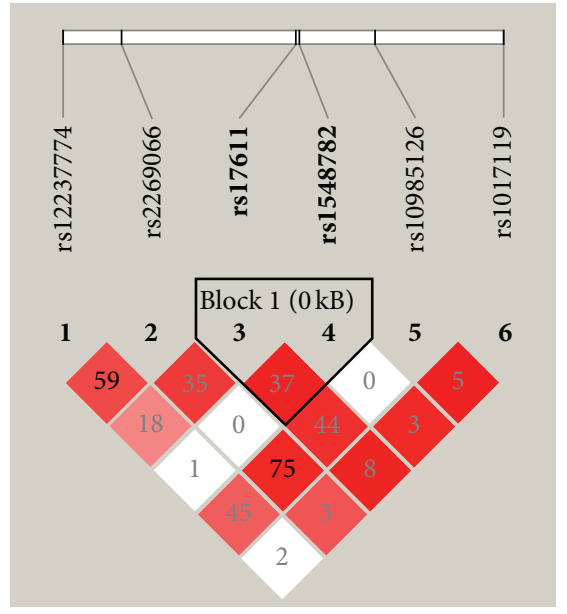

(c)

FIGURE 1: Linkage disequilibrium (LD) structure of the C5 locus for DR (a), NPDR (b), and PDR (c). LD was measured using data from all controls and total DR and its subtypes. The haplotype block was defined by the confidence interval method implemented in the Haploview software. The LD $\left(r^{2}\right)$ between any two SNPs is listed in the cross cells. DR: diabetic retinopathy; NPDR: nonproliferative diabetic retinopathy; PDR: proliferative diabetic retinopathy.

Among the 295 DR patients, 139 (47.1\%) were NPDR and $156(52.9 \%)$ were PDR; stratification analysis by the DR stage was performed. In PDR patients, significant lower frequencies of G allele and GG homozygosity for C5-rs17611 were found compared to that in DM group even after multiple testing correction $\left(P_{\text {corr }}=0.032, \mathrm{OR}=0.65,95 \% \mathrm{CI}=\right.$ 0.48-0.87; $P_{\text {corr }}=0.016, \mathrm{OR}=0.37,95 \% \mathrm{CI}=0.19-0.71$, resp.), implying a protective effect; such difference was not observed in NPDR patients (Table 3). For other SNPs, no significant differences in the allelic or genotypic frequencies were found in either NPDR or PDR subtypes compared with DM controls.

Pairwise LD analysis showed that two SERPING1 tagging SNPs were included in one haplotype block in NPDR,
PDR, and total DR patients. Three groups showed a similar distribution of haplotype. No haplotype was significantly associated with any group (all $P>0.1$, Table 4 ). Regarding C5, LD analysis revealed one haplotype block in three groups including SNPs rs17611 (the most significant finding) and rs1548782 (Figure 1). The haplotype AA, defined by the two SNPs, showed a significant risk for PDR patients $(P=0.004$, permutation $P=0.023$; $\mathrm{OR}=1.54,95 \% \mathrm{CI}=1.15-2.06)$. No significant haplotype association was detected among the other two comparisons (Table 5).

Considering the significance of C5-rs17611 in this study, correlations of the specific genotype with clinical features were evaluated in total DR patients. The results showed that DR patients carrying protective rs17611 GG genotype 


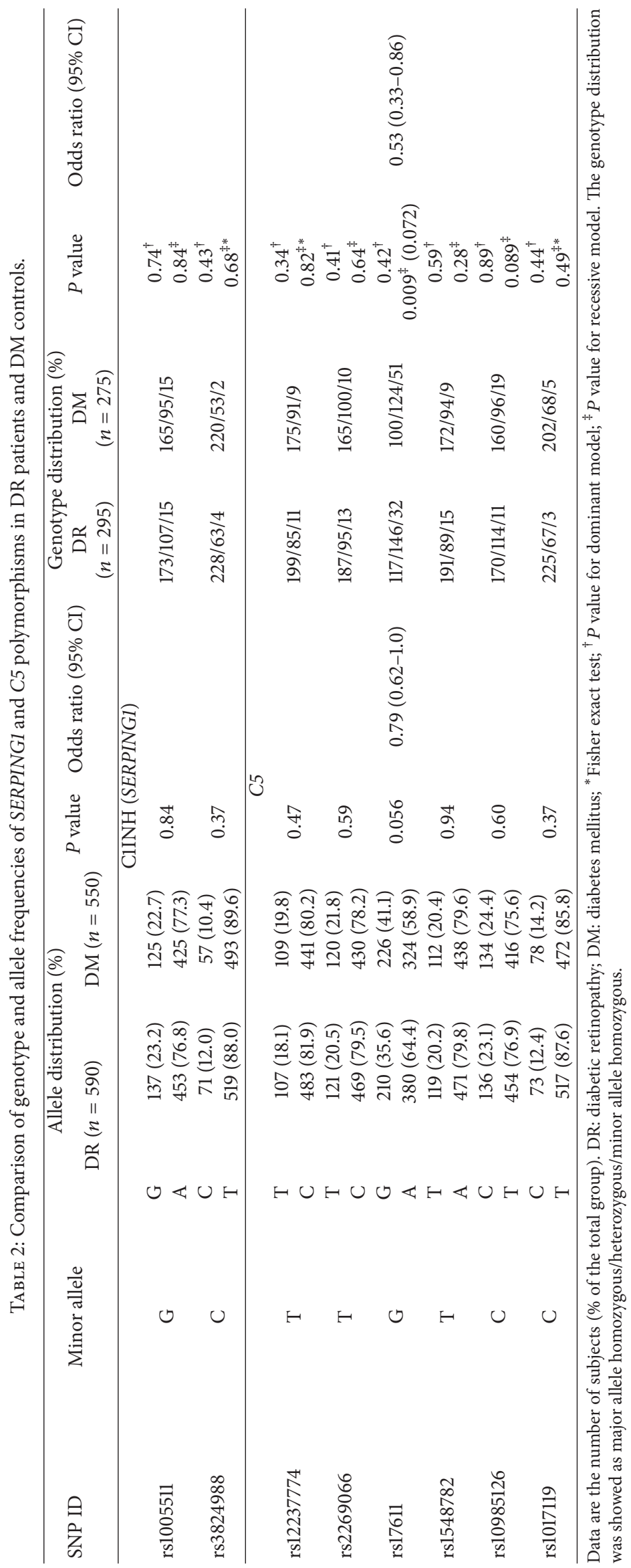


TABLE 3: Comparison of genotype and allele frequency of C5-rs17611 in DR and DM stratified by disease severity.

\begin{tabular}{|c|c|c|c|c|c|c|c|c|}
\hline \multirow{2}{*}{ SNP ID } & \multirow{2}{*}{ Genotype/allele } & \multirow{2}{*}{$\begin{array}{c}\text { NPDR } \\
(n=139)\end{array}$} & \multirow{2}{*}{$\begin{array}{c}\text { PDR } \\
(n=156)\end{array}$} & \multirow{2}{*}{$\begin{array}{c}\mathrm{DM} \\
(n=275)\end{array}$} & \multicolumn{2}{|c|}{ NPDR versus DM } & \multicolumn{2}{|c|}{ PDR versus DM } \\
\hline & & & & & $P$ value & $\begin{array}{l}\text { Odds ratio } \\
(95 \% \mathrm{CI})\end{array}$ & $P$ value & $\begin{array}{c}\text { Odds ratio } \\
(95 \% \mathrm{CI})\end{array}$ \\
\hline \multirow{5}{*}{ rs17611 } & GG & $20(14.4)$ & $12(7.7)$ & $51(18.5)$ & $0.51^{\dagger}$ & $\begin{array}{c}1.15 \\
(0.75-1.78)\end{array}$ & $0.062^{\dagger}$ & $\begin{array}{c}0.68 \\
(0.46-1.02)\end{array}$ \\
\hline & AG & $73(52.5)$ & $73(46.8)$ & $124(45.1)$ & $0.29^{\ddagger}$ & $\begin{array}{c}0.74 \\
(0.42-1.30)\end{array}$ & $\begin{array}{l}0.002^{\ddagger} \\
(0.016)\end{array}$ & $\begin{array}{c}0.37 \\
(0.19-0.71)\end{array}$ \\
\hline & AA & $46(33.1)$ & $71(45.5)$ & $100(36.4)$ & $0.62^{\#}$ & $\begin{array}{c}0.85 \\
(0.46-1.59)\end{array}$ & $\begin{array}{l}0.001^{\#} \\
(0.008)\end{array}$ & $\begin{array}{c}0.33 \\
(0.17-0.67)\end{array}$ \\
\hline & G & $113(40.6)$ & $97(31.1)$ & $226(41.1)$ & 0.90 & $\begin{array}{c}0.98 \\
(0.73-1.32)\end{array}$ & $\begin{array}{c}0.004 \\
(0.032)\end{array}$ & $\begin{array}{c}0.65 \\
(0.48-0.87)\end{array}$ \\
\hline & $\mathrm{A}$ & $165(59.4)$ & $215(68.9)$ & $324(58.9)$ & & & & \\
\hline
\end{tabular}

Data are the number of subjects (\% of the total group). NPDR: nonproliferative diabetic retinopathy; PDR: proliferative diabetic retinopathy; DM: diabetes mellitus. ${ }^{\dagger} P$ value for dominant model; ${ }^{\ddagger} P$ value for recessive model; ${ }^{\#} P$ value for codominant model.

TABLE 4: Haplotype association of SERPING1 gene with DR and its subtypes.

\begin{tabular}{|c|c|c|c|c|c|c|c|}
\hline \multirow{2}{*}{$\begin{array}{l}\text { Haplotype } \\
\text { rs1005511- } \\
\text { rs3824988 }\end{array}$} & \multicolumn{4}{|c|}{ Frequency } & \multicolumn{3}{|c|}{ Association $(P$ value $)$} \\
\hline & Total DR & NPDR & PDR & $\mathrm{DM}$ & $\begin{array}{c}\text { DR versus } \\
\text { DM }\end{array}$ & $\begin{array}{c}\text { NPDR versus } \\
\text { DM }\end{array}$ & $\begin{array}{c}\text { PDR versus } \\
\text { DM }\end{array}$ \\
\hline A-T & 0.76 & 0.79 & 0.74 & 0.77 & 0.79 & 0.51 & 0.31 \\
\hline G-T & 0.12 & 0.11 & 0.13 & 0.13 & 0.60 & 0.38 & 0.99 \\
\hline G-C & 0.12 & 0.10 & 0.13 & 0.10 & 0.42 & 0.96 & 0.19 \\
\hline
\end{tabular}

would present a delayed progression from DM to DR onset compared with patient carrying AA genotype $(9.3 \pm 6.4$ versus 7.0 $\pm 5.5, P=0.045$; Figure 2); no significant difference for other clinical features was detected among different genotype carriers.

\section{Discussion}

In this study, we performed a haplotype-tagging SNP analysis of two complement pathway genes, SERPING1 and C5, in T2DM and DR patients. Our results demonstrated that C5rs17611 was significantly associated with DR, particularly conferred to the PDR susceptibility; this functional variant also linked with certain clinical significance. Moreover, a haplotype conferring an increased risk for PDR was also detected. In contrast, none of the SNPs in SERPING1 were significantly associated with DR and its subtypes. These findings together suggest that SERPING1 is not a disease gene for DR, but C5 is likely to be a susceptibility gene for DR in Chinese patients. To our knowledge, this is the first genetic study to investigate SERPING1 and C5 genes in DR patients. Over the past decade, great achievements have been made in elucidating the genetic background of the disease; so far, more than 30 DR-associated genes involved in different metabolic mechanisms and functional pathways have been reported $[22,23]$. Our previous study has successfully identified two complement alternative pathway genes, $C F H$ and $C F B$, which were associated with DR development [18]. Results of this study enrich our knowledge of the genetic architecture of DR and the involvement of each complement pathway in DR pathogenesis. In addition, we also found that the proportions of insulin application were higher in DR group than that in DM controls; it was supposed that these DM patients without complications have a relatively good metabolic control, which may explain the lower frequency of individuals with insulin therapy.

As described above, the complement system is a key component of innate immunity, consisting of a large family of membrane-bound proteins that are critical for protection against bacterial infection and immune complex deposition. Uncontrolled complement activation is considered an important contributor in the pathogenesis of DR [15]. C5, being the first of many components of the terminal pathway, mediates many potent inflammatory events and plays a major role in the complement system. In the cascade, a critical event is the cleavage of C5 into fragments of C5a and C5b, as well as the subsequent formation of MAC (C5b-9) which is involved in cytolysis, cell activation, and production of inflammatory mediators [24]. In vitro study has revealed that C5a treatment induced increased production of several inflammatory cytokines, such as MCP-1, IL-6, IL-8, and VEGF, from retinal pigment epithelial cells $[25,26]$. In clinical study, C5b-9 deposition was detected on the endothelial surface of retinal vessels in eye donors with diabetes; vitreous concentration of C5a increased significantly in PDR patients compared with controls $[14,27]$. Furthermore, C5 gene has been found to affect susceptibility to several inflammatory conditions, including AMD, rheumatoid arthritis, and renal allograft outcomes [28-30]. In the present study, C5-rs17611 was found to be associated with PDR patients; meanwhile, rs17611 was also found to be associated with periodontitis and the GG genotype was linked with increased C5 levels 
TABLE 5: Haplotype association of C5 gene with DR and its subtypes.

\begin{tabular}{lcccccc}
\hline $\begin{array}{l}\text { Haplotype } \\
\text { rs17611- } \\
\text { rs1548782 }\end{array}$ & Total DR & NPDR & PDR & DM & $\begin{array}{c}\text { Association (P value) (permutation test) } \\
\text { DR versus } \\
\text { DM }\end{array}$ & $\begin{array}{c}\text { NPDR versus } \\
\text { PDR versus } \\
\text { DM }\end{array}$ \\
\hline A-A & 0.64 & 0.59 & 0.69 & 0.59 & 0.064 & 0.94 \\
G-T & 0.20 & 0.23 & 0.17 & 0.20 & 0.88 & 0.29 \\
G-A & 0.16 & 0.17 & 0.15 & 0.21 & 0.025 & 0.21 \\
\end{tabular}

NPDR: nonproliferative diabetic retinopathy; PDR: proliferative diabetic retinopathy; DM: diabetes mellitus. $P_{\text {corr }}$ association analysis results from permutation test (iterations 10,000).

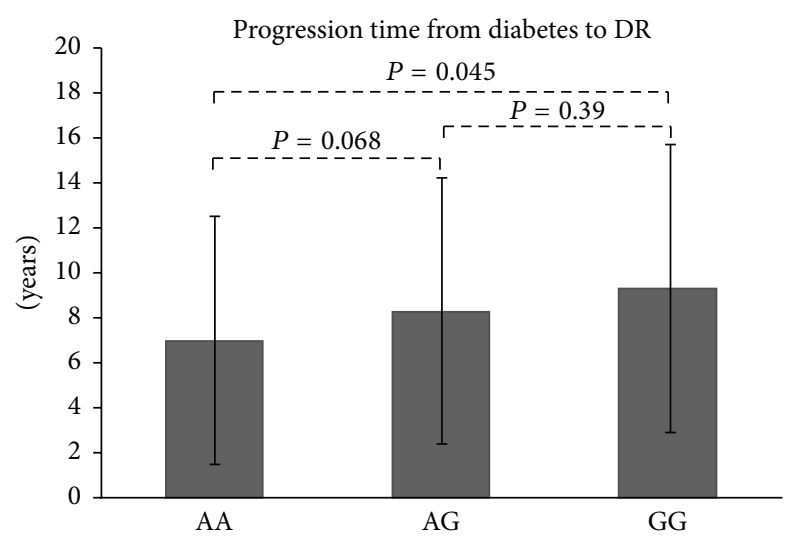

FIGURE 2: The average time of progression from diabetes to DR according to genotype. AA: $7.0 \pm 5.5$; AG: $8.3 \pm 5.9$; GG: $9.3 \pm 6.4$ (years).

in patients with rheumatoid arthritis [29, 31]. The change of rs17611 A>G nucleotide results in the synthesis of Valine instead of Isoleucine; a functional analysis on rs17611 showed that individuals homozygously expressing the risk s17611 allele exhibit increased C5a and decreased C5 in plasma, evidence of increased C5 turnover; this structural change might alter the rate of $\mathrm{C} 5$ cleavage and explain its association with inflammatory diseases [29].

Component 1 inhibitor gene (SERPING1) encoding $\mathrm{CIINH}$ is a key regulator in classic and lectin complement pathway and involved in the development of several immunerelated diseases. In addition, CIINH was also found to be expressed in both retinal and retinal pigment epithelium (RPE) layers [32-34]. Based on the evidence, SERPING1 was considered as a candidate gene for DR. But, in our study, no association between SERPING1 polymorphisms and DR was found, even stratified by DR stage or considered clinical features. The results suggested that SERPING1, and the geneinvolved classical pathway, might not contribute significantly to the risk of DR. Further studies to determine the biologic roles of these polymorphisms and the haplotype in DR are still warranted; additionally, it would be better to include healthy controls in this study to fully reflect the disease association.

In summary, this study first demonstrated that $C 5$ rs17611 is a susceptibility locus for DR and particularly predisposes to
PDR subtype with clinical significance. The complement classical pathway gene, SERPING1, may confer no or limited risk for DR development. Together with our previous findings, our results help to further enrich the growing understanding of genetic spectrum of DR and clarify the involvement of each complement pathway in DR pathogenesis from molecular perspectives.

\section{Conflict of Interests}

The authors declare that they have no conflict of interests.

\section{Authors' Contribution}

Ming Ming Yang, Jiao Jie Fan, and Yan Teng designed the experiments. Jun Wang and Jiao Jie Fan performed the experiments. Ming Ming Yang, Yun Duan Sun, and Jun Wang performed the analysis and wrote the paper. Yan Bo Li and Hong Ren revised the paper. All authors contributed to the editing of the paper and to scientific discussions. Ming Ming Yang and Jun Wang contributed equally to this work. Dr. Ming Ming Yang and Dr. Jiao Jie Fan contributed equally to the work in this paper and can be considered as cocorresponding authors.

\section{Acknowledgments}

The authors express their greatest gratitude to all the participants in this study. This study was supported in part by the National Natural Science Foundation of China (Grant no. 81500711); Natural Science Foundation of Heilongjiang Province of China (Grant no. QC2015094); Science and Research Foundation under the Health Committee of Heilongjiang Province (Grant no. 2014-442); and Novo Nordisk China Diabetes Young Scientific Talent Research Funding.

\section{References}

[1] Z. He and G. L. King, "Microvascular complications of diabetes," Endocrinology and Metabolism Clinics of North America, vol. 33, no. 1, pp. 215-238, 2004.

[2] I. Prokopenko, M. I. McCarthy, and C. M. Lindgren, "Type 2 diabetes: new genes, new understanding," Trends in Genetics, vol. 24, no. 12, pp. 613-621, 2008. 
[3] R. N. Frank, "Diabetic retinopathy," The New England Journal of Medicine, vol. 350, no. 1, pp. 48-58, 2004.

[4] M. S. Roy, "Diabetic retinopathy in African Americans with type 1 diabetes: the New Jersey 725: II. Risk factors," Archives of Ophthalmology, vol. 118, no. 1, pp. 105-115, 2000.

[5] M. Rema, G. Saravanan, R. Deepa, and V. Mohan, "Familial clustering of diabetic retinopathy in South Indian Type 2 diabetic patients," Diabetic Medicine, vol. 19, no. 11, pp. 910-916, 2002.

[6] N. H. Arar, B. I. Freedman, S. G. Adler et al., "Heritability of the severity of diabetic retinopathy: the FIND-Eye study," Investigative Ophthalmology and Visual Science, vol. 49, no. 9, pp. 3839-3845, 2008.

[7] M. Murea, L. Ma, and B. I. Freedman, "Genetic and environmental factors associated with type 2 diabetes and diabetic vascular complications," Review of Diabetic Studies, vol. 9, no. 1, pp. 6-22, 2012.

[8] S.-I. Yamagishi and T. Matsui, "Advanced glycation end products (AGEs), oxidative stress and diabetic retinopathy," Current Pharmaceutical Biotechnology, vol. 12, no. 3, pp. 362-368, 2011.

[9] M. I. Lopez Galvez, "Protein kinase C inhibitors in the treatment of diabetic retinopathy. Review," Current Pharmaceutical Biotechnology, vol. 12, no. 3, pp. 386-391, 2011.

[10] S. Wang, J. K. Park, and E. J. Duh, "Novel targets against retinal angiogenesis in diabetic retinopathy," Current Diabetes Reports, vol. 12, no. 4, pp. 355-363, 2012.

[11] A. M. A. El-Asrar, "Role of inflammation in the pathogenesis of diabetic retinopathy," Middle East African Journal of Ophthalmology, vol. 19, no. 1, pp. 70-74, 2012.

[12] A. M. Joussen, V. Poulaki, M. L. Le et al., "A central role for inflammation in the pathogenesis of diabetic retinopathy," The FASEB Journal, vol. 18, no. 12, pp. 1450-1452, 2004.

[13] M. Tomic, S. Ljubic, and S. Kastelan, "The role of inflammation and endothelial dysfunction in the pathogenesis of diabetic retinopathy," Collegium Antropologicum, vol. 37, supplement 1, pp. 51-57, 2013.

[14] J. Zhang, C. Gerhardinger, and M. Lorenzi, "Early complement activation and decreased levels of glycosylphosphatidylinositolanchored complement inhibitors in human and experimental diabetic retinopathy," Diabetes, vol. 51, no. 12, pp. 3499-3504, 2002.

[15] J. Østergaard, T. K. Hansen, S. Thiel, and A. Flyvbjerg, "Complement activation and diabetic vascular complications," Clinica Chimica Acta, vol. 361, no. 1-2, pp. 10-19, 2005.

[16] B.-B. Gao, X. Chen, N. Timothy, L. P. Aiello, and E. P. Feener, "Characterization of the vitreous proteome in diabetes without diabetic retinopathy and diabetes with proliferative diabetic retinopathy," Journal of Proteome Research, vol. 7, no. 6, pp. 2516-2525, 2008.

[17] M. García-Ramírez, F. Canals, C. Hernández et al., "Proteomic analysis of human vitreous fluid by fluorescence-based difference gel electrophoresis (DIGE): a new strategy for identifying potential candidates in the pathogenesis of proliferative diabetic retinopathy," Diabetologia, vol. 50, no. 6, pp. 1294-1303, 2007.

[18] J. Wang, M. M. Yang, Y. B. Li, G. D. Liu, Y. Teng, and X. M. Liu, "Association of CFH and CFB gene polymorphisms with retinopathy in type 2 diabetic patients," Mediators of Inflammation, vol. 2013, Article ID 748435, 7 pages, 2013.

[19] G. Bajic, S. E. Degn, S. Thiel, and G. R. Andersen, "Complement activation, regulation, and molecular basis for complementrelated diseases," The EMBO Journal, vol. 34, no. 22, pp. 27352757, 2015.
[20] K. G. M. M. Alberti and P. Z. Zimmet, "Definition, diagnosis and classification of diabetes mellitus and its complications. Part 1: diagnosis and classification of diabetes mellitus. Provisional report of a WHO consultation," Diabetic Medicine, vol. 15, no. 7, pp. 539-553, 1998.

[21] Early Treatment Diabetic Retinopathy Study Research Group, "Grading diabetic retinopathy from stereoscopic color fundus photographs-an extension of the modified Airlie House classification. ETDRS report number 10. Early Treatment Diabetic Retinopathy Study Research Group," Ophthalmology, vol. 98, no. 5, supplement, pp. 786-806, 1991.

[22] E. Ahlqvist, N. R. van Zuydam, L. C. Groop, and M. I. McCarthy, "The genetics of diabetic complications," Nature Reviews Nephrology, vol. 11, no. 5, pp. 277-287, 2015.

[23] C. L. Hanis and D. M. Hallman, "Genetics of diabetic retinopathy," Current Diabetes Reports, vol. 6, no. 2, pp. 155-161, 2006.

[24] D. Turnberg and M. Botto, "The regulation of the complement system: insights from genetically-engineered mice," Molecular Immunology, vol. 40, no. 2-4, pp. 145-153, 2003.

[25] D. N. Cortright, R. Meade, S. M. Waters, B. L. Chenard, and J. E. Krause, "C5a, but not C3a, increases VEGF secretion in ARPE19 human retinal pigment epithelial cells," Current Eye Research, vol. 34, no. 1, pp. 57-61, 2009.

[26] Y. Fukuoka, M. Strainic, and M. E. Medof, "Differential cytokine expression of human retinal pigment epithelial cells in response to stimulation by C5a," Clinical and Experimental Immunology, vol. 131, no. 2, pp. 248-253, 2003.

[27] D. Muramatsu, Y. Wakabayashi, Y. Usui, Y. Okunuki, T. Kezuka, and H. Goto, "Correlation of complement fragment C5a with inflammatory cytokines in the vitreous of patients with proliferative diabetic retinopathy," Graefe's Archive for Clinical and Experimental Ophthalmology, vol. 251, no. 1, pp. 15-17, 2013.

[28] D. C. Baas, L. Ho, S. Ennis et al., "The complement component 5 gene and age-related macular degeneration," Ophthalmology, vol. 117, no. 3, pp. 500-511, 2010.

[29] J. L. Giles, E. Choy, C. van den Berg, B. P. Morgan, and C. L. Harris, "unctional analysis of a complement polymorphism (rs17611) associated with rheumatoid arthritis," The Journal of Immunology, vol. 194, no. 7, pp. 3029-3034, 2015.

[30] J. C. Jeong, Y.-H. Hwang, H. Kim et al., "Association of complement 5 genetic polymorphism with renal allograft outcomes in Korea," Nephrology, Dialysis, Transplantation, vol. 26, no. 10, pp. 3378-3385, 2011.

[31] L. Chai, Y.-Q. Song, K.-Y. Zee, and W. K. Leung, "Single nucleotide polymorphisms of complement component 5 and periodontitis," Journal of Periodontal Research, vol. 45, no. 3, pp. 301-308, 2010.

[32] S. Ennis, C. Jomary, R. Mullins et al., "Association between the SERPING1 gene and age-related macular degeneration: a twostage case-control study," The Lancet, vol. 372, no. 9652, pp. 1828-1834, 2008.

[33] A. E. Davis III, K. Aulak, R. B. Parad et al., "C1 inhibitor hinge region mutations produce dysfunction by different mechanisms," Nature Genetics, vol. 1, no. 5, pp. 354-358, 1992.

[34] D. E. Madsen, J. J. Sidelmann, D. Biltoft, J. Gram, and S. Hansen, "C1-inhibitor polymers activate the FXII-dependent kallikreinkinin system: implication for a role in hereditary angioedema," Biochimica et Biophysica Acta (BBA)-General Subjects, vol. 1850, no. 6, pp. 1336-1342, 2015. 


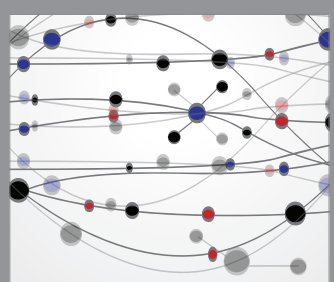

The Scientific World Journal
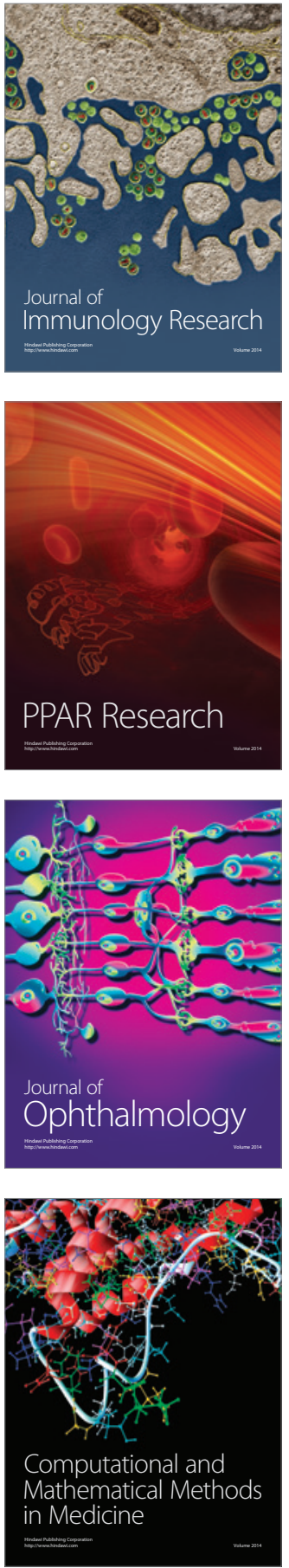

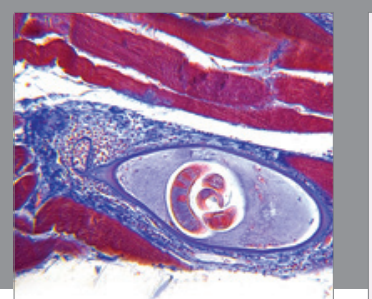

Gastroenterology Research and Practice

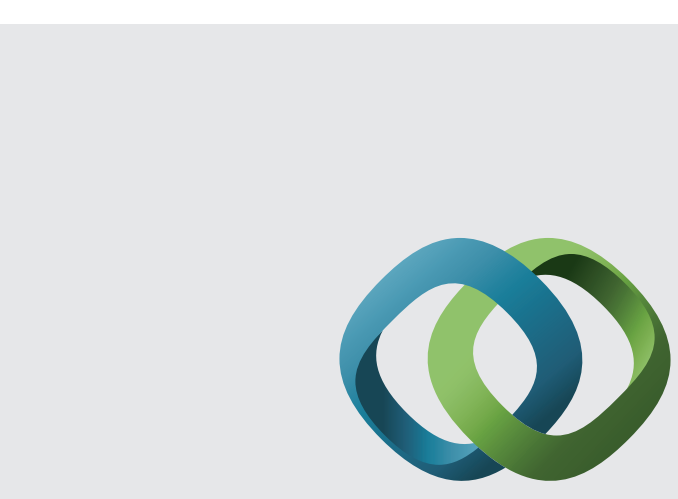

\section{Hindawi}

Submit your manuscripts at

http://www.hindawi.com
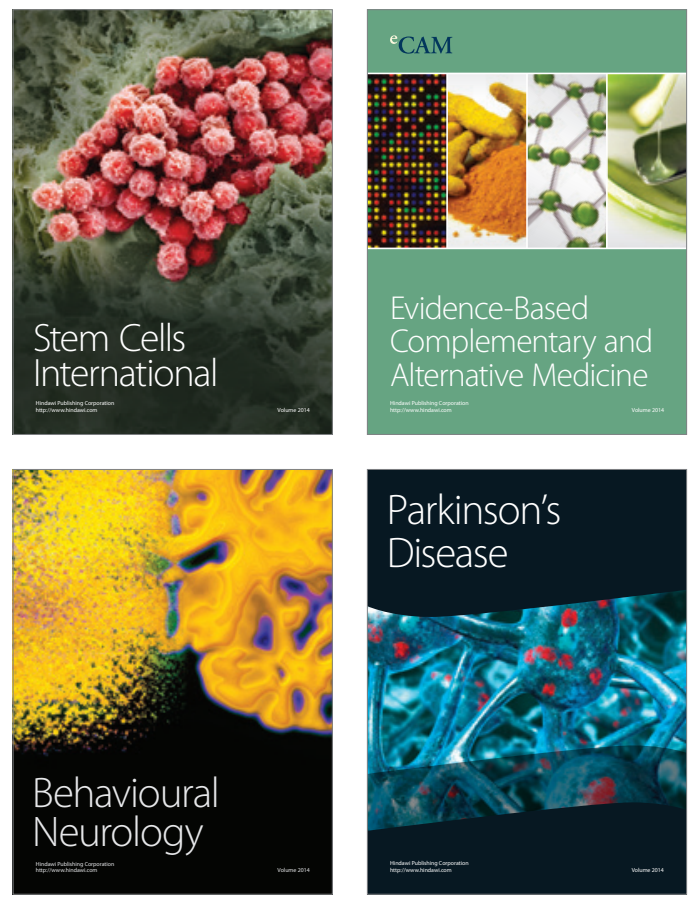
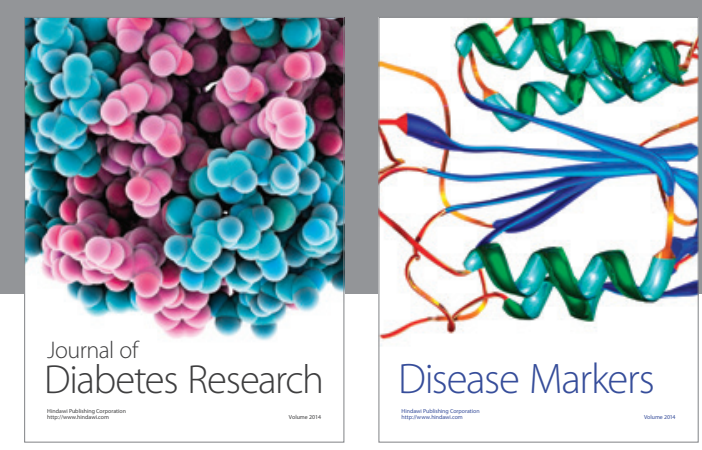

Disease Markers
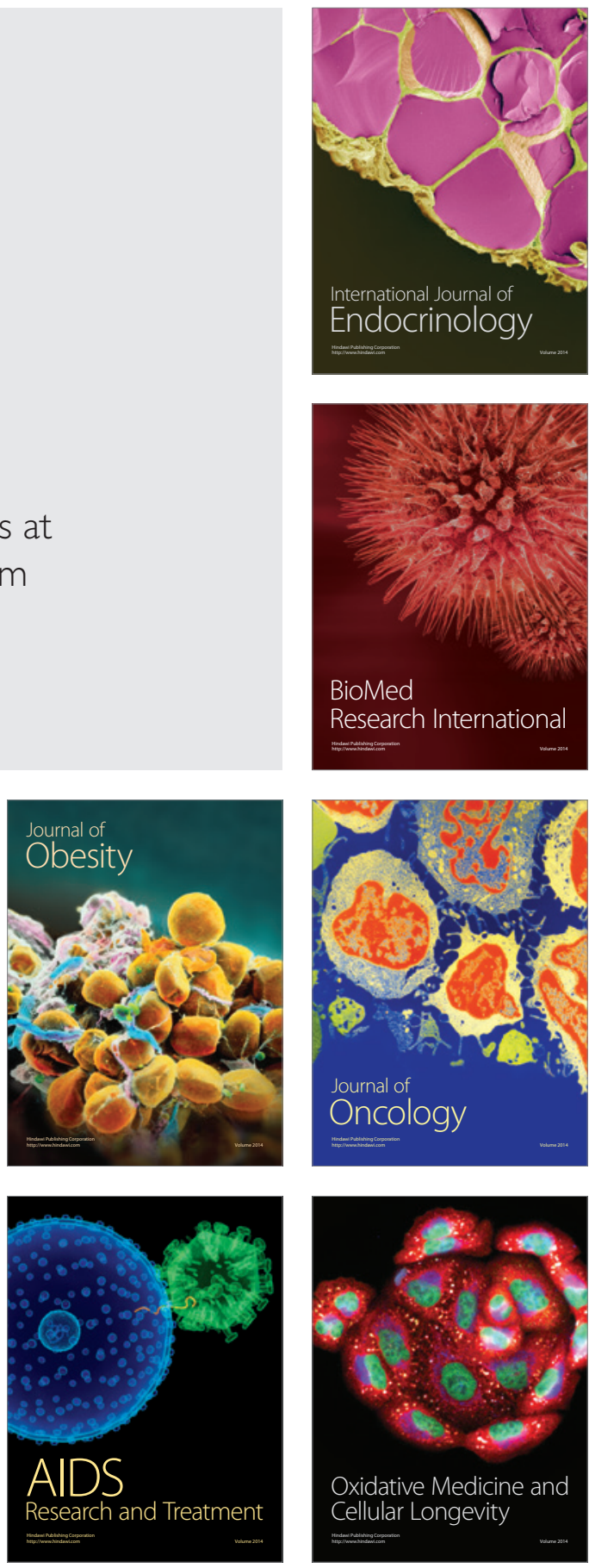